\title{
An experimental investigation of viscosity of a newly developed natural polymer-based media for abrasive flow machining (AFM) of 3D printed ABS parts
}

DOI:10.36909/jer.13643

\author{
" Abdul Wahab Hashmi ${ }^{\mathrm{a}}$, Harlal Singh Mali ${ }^{\mathrm{b}}$, Anoj Meena ${ }^{\mathrm{c}}, \mathrm{a}^{*}$ \\ "abc Mechanical Engineering Department, Malaviya National Institute of Technology, Jaipur- \\ 302017, India"
}

\begin{abstract}
Abrasive Flow Machining (AFM) is the method of finishing complex surfaces and internal channels with the help of extrusion pressure and abrasive-laden viscoelastic polymer media. This paper is based on developing a new AFM media using a natural waste polymer as a base material. In the article, a natural polymer media viz. rice husk ash-based media has been developed, and subsequently, rheological analysis has been done, and experimentation has been performed on Anton-paar® rheometer to optimize the viscosity of these newly developed AFM media. In this research study, the hollow elliptical shape of ABS (acrylonitrile-butadiene-styrene) material was manufactured using the FDM technique and then finished with a one-way AFM machine. This paper examined the parametric dependencies of AFM process parameters on finishing FDM printed hollow elliptical parts. The improved surface roughness of the FDM printed hollow elliptical parts has been investigated relating to the AFM process parameters. The maximum surface roughness has been achieved by $95.98 \%$.
\end{abstract}

Corresponding Author-hashmicad@gmail.com

Keywords: Abrasive Flow Machining (AFM), AFM Media, Viscosity, Viscometer, FDM Printed Parts, One-way AFM machine.

\section{Introduction}

For the last few decades, industries have been utilizing conventional and non-traditional finishing procedures, such as honing, lapping, and grinding, etc., to acquire the desired finishing of the machining elements. However, these conventional methods are restricted to be done on some particular geometries and are non-applicable to intricate geometric profiles to do high-level finishing, which is desired during the process. Limitations of the conventional finishing approaches lead to the production of progressive and developed finishing procedures, such as Abrasive Flow Machining (AFM) (Kumar and Hiremath, 2016). 
AFM is considered to be the non-traditional procedure having extensive applications in surface refinement, precision deburring, radioing, as well as amputation of recast layer (Perry and Stackhouse, 1989; Sudhakara, Suresh, and Vinod, 2020). AFM has the potential to efficiently polish the intricate geometries that are difficult to access. The process of AFM comprises the tooling, machining, as well as abrasive media (Rhoades, 1991), where the machine possesses two media cylinders placed opposite to each other and are processed via "power pack assembly." Tooling crutches workpiece strongly at the time of the machining process and makes the "abrasive media area" to be complete on time. Furthermore, abrasive media, which is the amalgamation of abrasive particles and polymer-based, is utilized as the finishing tool (Singh and Sankar, 2019). Here, in this process, the abrasive media is forced out via a small restricted passage present between the tooling and workpiece, resulting in work surface abrasion (Sankar et al., 2019). The material removal is done using three distinct modes such as micro-plowing, free rubbing, and micro-cutting (Kum et al., 2020; Dixit, Sharma, and Kumar, 2021c). The new surfaces in the abrasive machining process are created by random attacks of the abrasive elements over the irregularities that lie on the work surface (Walia, Shan, and Kumar, 2009; Ali et al., 2020). The extrusion pressure that is applied using the hydraulic cylinders generates the normal force as well as the "axial force" on the abrasive elements. Furthermore, the normal force pierces the abrasive over the workforce while the axial element offers the axial movement to that of the penetrated abrasive and eradicates the substance from that of the work surface (Lin et al., 2020). In ductile materials, "microploughing" and micro-cutting phenomena occur together; however, the micro-cutting process is considered to be more prevailing in bitter materials. Parametric analysis and study of the AFM process are considered to be the most substantial domain of exploration in AFM. This process of AFM responses such as micro-hardness, surface finishing, material removal, wettability, as well, as residual stress is impacted by several inter-dependent factors and parameters such as "media-based," "machine-based," or "variant-based." The media-based parameters are done more commonly in AFM and its various variants. Jayakumar et al. have investigated the mechanical properties of 3D printed PLA parts. The authors have analyzed the effect of printing process parameters effect i.e.infill, density $(\%)$, Nozzle temperature $\left({ }^{\circ}\right)$, and print orientation ( $\mathrm{N}$ et al., 2021). Mohamed et al. have investigated the effect of layer thickness, infill density, and build orientation on fabrication time and material consumption during the fused deposition modeling process. The authors have optimized the process parameters using the response surface methodology (RSM) technique (Mohamed et al., 2015). Kumar et al. have analyzed the tribological behavior of 3D printed ABS parts using a 
pin on disc wear set-up. The authors have optimized the 3D printing process parameters for minimum wear conditions using the RSM technique (Kumar et al., 2021). (Arivazhagan et al., 2021) have used 3d printed patient-specific bone models of leg and ankle foot from medical images by processing "Digital Imaging and Communications in Medicine (DICOM)" files for anatomy education. The authors have $3 \mathrm{~d}$ printed the bone model using ABS material from the $3 \mathrm{D}$ model obtained from the image processing.

The stair-stepping/stair-casing effect is the most common phenomenon that occurs during layer manufacturing, i.e., additive manufacturing process, as shown in figure 1, which causes the surface roughness in the additively manufactured parts. The various researchers proposed post-processing techniques to avoid these effects and improve the surface quality of additively manufactured parts (A.W. Hashmi et al. 2020).
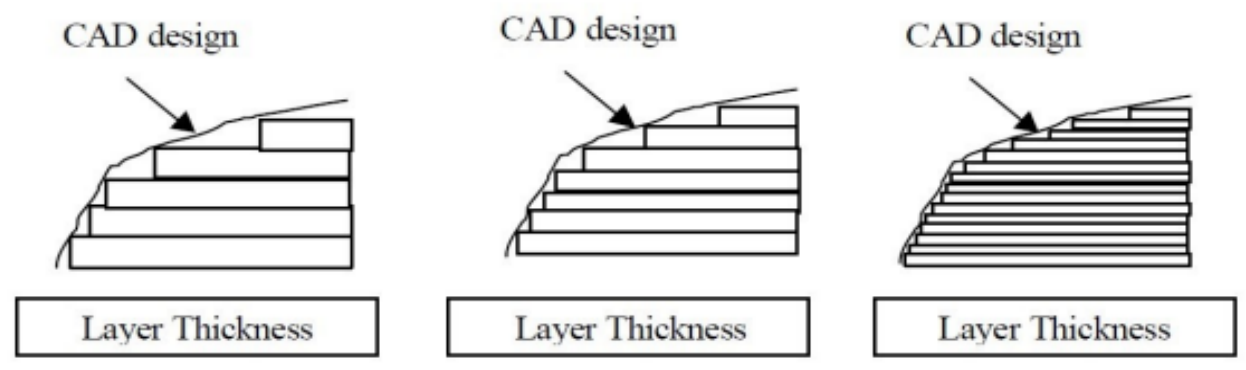

Figure 1. Stair-stepping/Stair-casing effect occurs in the additive manufacturing process.

\subsection{AFM Media Development}

Baraiya et al. (Baraiya et al., 2020) utilized silicone rubber having additives along with pure silicone as base material to create the abrasive media. It was found that silicone rubber having the additives mixed in it possesses higher viscosity as compared to pure silicone and provided a good finishing performance. Authors (Dixit, Sharma, and Kumar, 2021c) have claimed that abrasive media is composed of Polyboronorgano compound hydrocarbon oil as well as metallic soap gel. Here the polymer was ready by reacting silicon oil with that of boron element within the catalyst of Lewis acid, and then it was heated. Here, the Ammonium carbonate was utilized for acid neutralization. In the final step, abrasive media was ready by combining the abrasives, gel, and polymer together in a specific amount. Rajesha et al. (Rajesha et al., 2010) created a "natural polymer-based abrasive media" that was of low cost and was sustainable. Natural polymer-based media comprised naphthenic processing oil, an 
ester-based polymer, and silicon carbide abrasive. "FTIR spectrum" presented that polymer media possesses the desired alkenes and that of the ester group explained the "elastic nature" of the polymer media. The thermal investigation showed that media of natural polymer had the potential to bear a temperature of around $71^{\circ} \mathrm{C}$, which was very high than the actual working range of AFM. In (Dabrowski, Marciniak, and Szewczyk, 2006), Dabrowski et al. developed the electrochemical variation of AFM by joining the AFM and ECM processes. To acquire the electrochemical effect, the authors developed and used the electrolytic abrasive media. The conventional polymer, in this case, was replaced by "poly (glycol and ethylene glycol)" that also behaved as the "electrolytic polymer." These polymers transport the abrasive elements and alleviate the material from that of work material owing to anodic dissolution. In (Jha and Jain, 2004), authors have generated the MRAFF process by merging AFM and MRF processes. Magnetorheological finishing operation has been developed for the MRAFF process by making use of mineral oil, grease, SiC, and CIP. Here, CIP took the shape of the columnar structure with an "applied magnetic field" and assisted in regulating the media rheology. The developed media acted as the "Newtonian fluid" without the magnetic field and as "Bingham plastic" with the magnetic field. Hydrogels possess the 3D cross-linked structure that is designed for the cross-linked polymer chain. They hold a huge amount of water and are hydrophilic (Dixit, Sharma and Kumar, 2021b, 2021a). Here, the author has investigated the thickening conformation of the guar gum, xanthan gum as well as starch. The viscosity of these components was marked as better than that of the individual elements/ constitutes. A guar-gum-based media for the AFM procedure has been developed in (Wei, Gao, and Wang, 2019), where the mixture of boric acid and borax was used for the media preparation. This paper presents the experimental investigation of a viscosity of newly developed natural polymer-based media to finish the FDM printed hollow elliptical surface using a one-way AFM process. The newly developed AFM polishing media uses rice husk ash as a base material, waste vegetable oil as a carrier liquid, gel (aloe barbadensis mill,cyamopsis tetragonoloba powder, and glycerin ) as additives. The AFM media viscosity and AFM process parameters have been investigated and selected for maximum improvement of material removal rate $(M R R)$ and surface roughness $(\Delta R a)$. The researchers have investigated the mechanical properties of briquette prepared using rice and micrometer-sized carbon particles from potato and yam skins for sustainable utilization of organic waste materials (Anggraeni et al., 2021). For the AFM process, there occurs the development of several abrasive media as shown in figure 2, which are described below: 


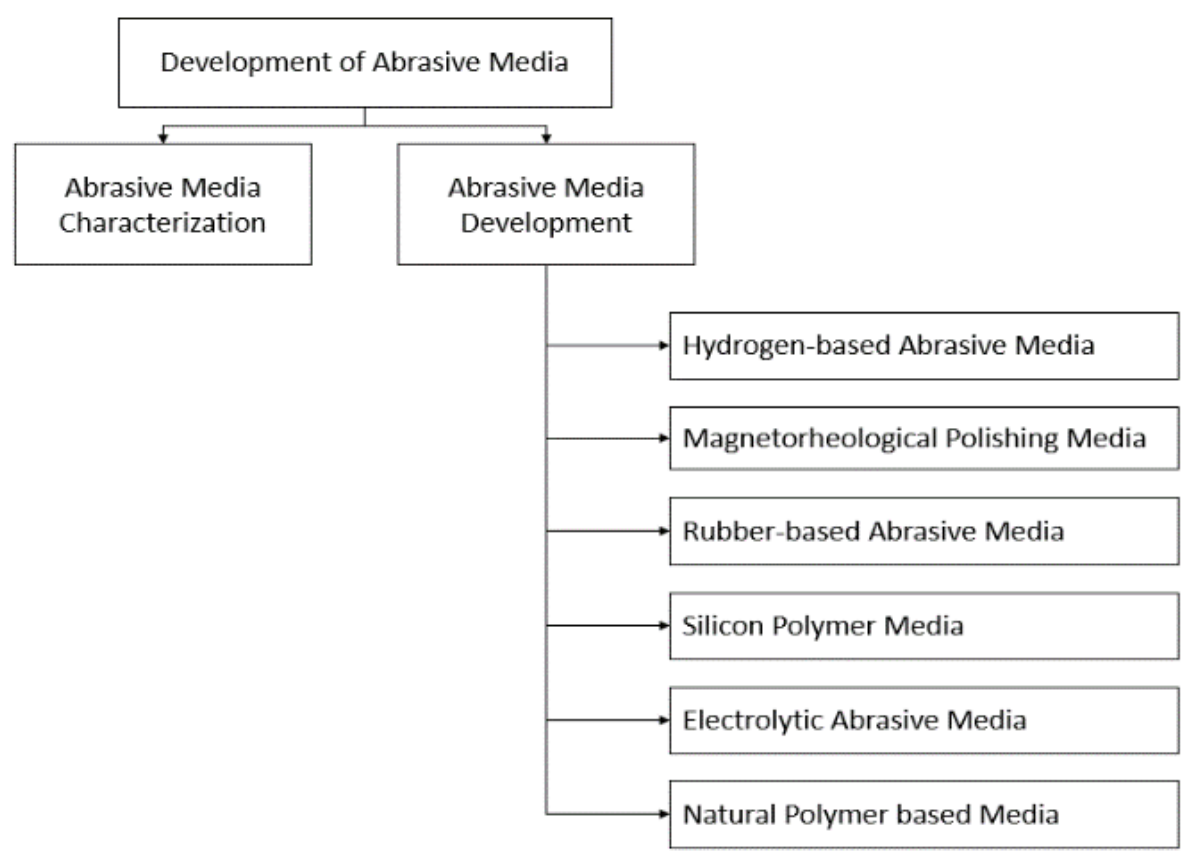

Figure. 2. Development of Abrasive Media

\section{Materials and Methods}

A hollow elliptical shape is designed with $50 \mathrm{~mm}$ length, $19.8 \mathrm{~mm}$ inlet diameter, and 7.2 mm outlet diameter using Autodesk ${ }^{\circledR}$ fusion360 Software. After designing the hollow truncated cone, It is 3D printed with acrylonitrile butadiene styrene (ABS) material using an FDM machine. CAD model and cross-sectional view of hollow elliptical design are shown in figure 3. The hollow elliptical shape was fabricated using the "Aha 3D" manufactured desktop 3D printer of the Protocenter 999 model, as shown in figure 4 (a). The samples of hollow elliptical shapes fabricated using the above-mentioned 3D printer are shown in figure 4 (b) - design and fabrication of one-way AFM. The AFM fixture has been designed to hold and support the hollow truncated cone shape using Autodesk® fusion360. The AFM fixture is designed in two parts: the top fixture and the bottom fixture, as shown in figure 5 (a). This fixture is also fabricated using "Aha 3D" desktop 3d printer of the Protocenter 999 model, as shown in figure 5 (b). The one-way AFM machine has been employed to finish the FDM printed hollow truncated cone's internal surface. The used one-way machine set-up for finishing operation is shown in figure 6. 


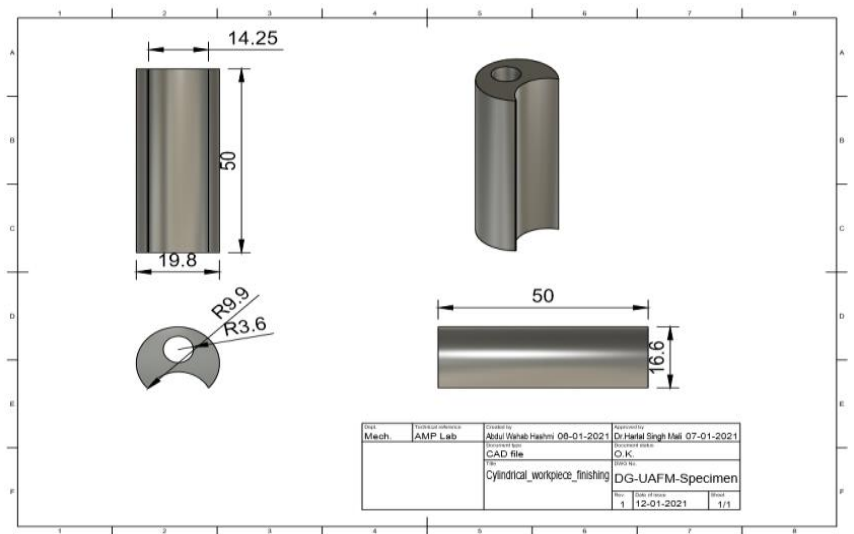

Figure 3. CAD model of the hollow elliptical shape.

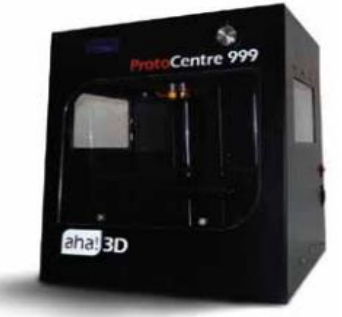

(a)

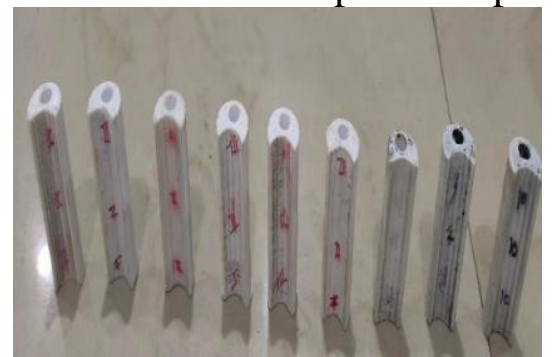

(b)

Figure 4. (a) "Aha 3D" desktop 3d printer of Protocenter 999 model (b) FDM printed samples of the hollow elliptical shape.

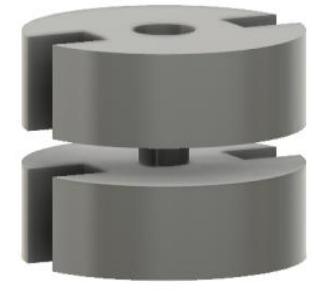

(a)

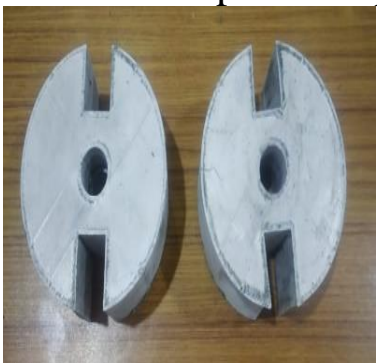

(b)

Figure 5. (a) Assembly of one-way AFM fixture, (b) FDM printed one-way AFM fixture.

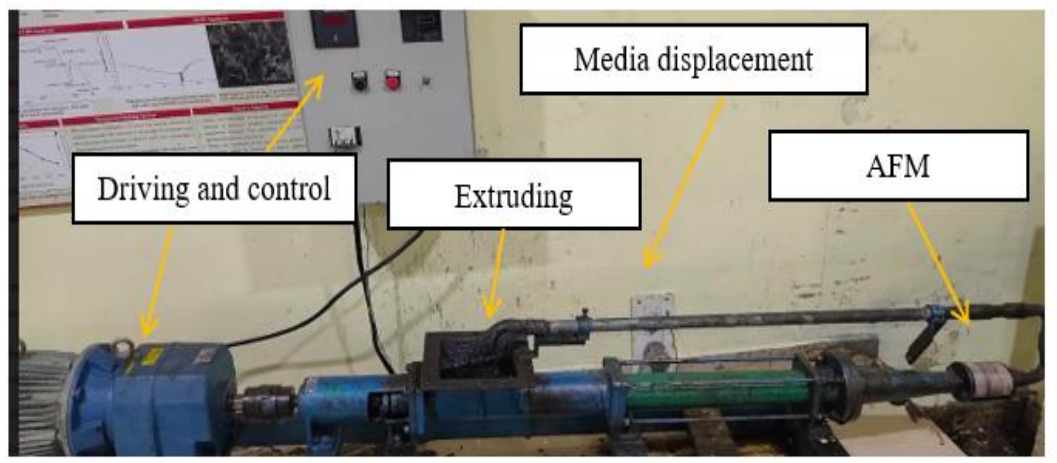

Figure 6. one-way AFM machine set-up.

\section{Development of Environmentally friendly and sustainable AFM (EASAFM) media}

The significant factor that can influence the finishing in the AFM process is abrasive polishing media. In this experiment, a new environment-friendly AFM media have been 
developed using a rice husk ash as base material, waste vegetable oil as liquid synthesizer, gel (aloe barbadensis mill,cyamopsis tetragonoloba powder, and glycerin ) as an additive, silicon carbide ( $\mathrm{SiC}$ ) as the abrasive. This newly developed AFM media is known as EFSAFM. The gel has low flow property and does not stick on the workpiece surface after contact so that the suitable abrasive media in AFM and the workpiece are polished consistently utilizing this kind of gel.

\section{Selection of media Base and additives}

A base material abrasive and additive is mixed in the AFM media, separately by varying abrasive and liquid synthesizer concentrations. Those all possible media bases are base material as a rice husk ash, waste vegetable oil as liquid synthesizer (\%30, \%40, \%50), SiC as abrasive particles (\%120,\%220,\%320), and gel (Aloe Barbadensis Mill, Cyamopsis Tetragonoloba Powder and glycerin) as additives.

\section{Procedure}

The $\mathrm{SiC}$ abrasive is blended in the gel by a blender machine. So as to get a consistently blended media, waste vegetable oil added to the gel in the process begins. Finally, the abrasive media is placed into the hopper, and the workpiece is fixed in the tooling and machined utilizing the alternating back and forth cycles through the tooling.

\section{Synthesis Method}

The media composition at that time it is challenging to measure the percentage of all content that is mixed on it. The selected media composition one by one and varying the concentration of all the contents for exact concentration. This selection is based on changing the media property, abrasive concentration, viscosity of media. 
The first step here is to measure all the quantities needed based on the abrasive concentration and take rice husk ash powder and gel mixing through a mixer machine. Now the base material is ready to mix $\mathrm{SiC}$ abrasive and waste vegetable oil liquid synthesizer with rice husk ash powder and gel. The steps of synthesis are shown in figures 7 and 8 .

\begin{tabular}{|ll|} 
Step & - Measure required amount of rice husk ash. \\
$\mathbf{1}$ & - Put measured quantity into bowl \\
Step & - Measure gel \\
$\mathbf{2}$ & - Mix With rice husk ash. \\
Step & - Measure required amount of waste vegetable oil. \\
3 & - Mix with step 2 mixture. \\
Step & - Measured abrasive (silicon carbide $\% 120, \% 220, \% 320)$. \\
4 & - Mix with step 3 mixture. \\
\hline
\end{tabular}

Figure. 7. A synthesis method of AFM media (Rice husk ash $+\mathrm{Gel}+\mathrm{Oil}+\mathrm{SiC}$ $\% 120, \% 220, \% 320)$.
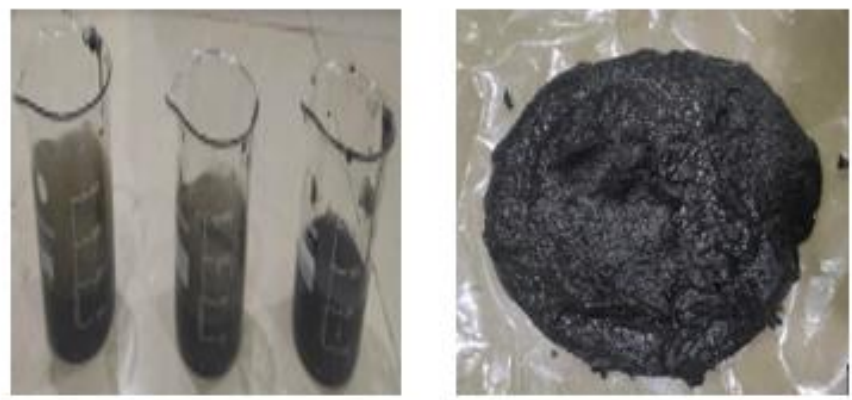

Figure 8. Rice husk ash-based waste natural polymer-based media Samples.

\section{Design of experiments and optimization of media variables for rheology study}

AFM Media is prepared by mixing rice husk ash as base material, waste vegetable oil (groundnut oil) as carrier medium, and gel (Aloe Barbadensis Mill, Cyamopsis Tetragonoloba powder, and glycerin) as additives. The rice husk ash-based AFM finishing media were prepared to optimize the media viscosity for finishing FDM printed ABS parts. While preparing the AFM media, three factors were considered: $\%$ abrasive concentration, Abrasive mesh size, and \% liquid synthesizer with respect to Taguchi L9 orthogonal experimental design. The percentage of abrasive concentration as 33, 50, 67, Abrasive mesh 
size as $120,220,320$, and percentage of the liquid synthesizer as $30,45,60$, as shown in figure 9. Process levels and parameters for the experimental design are shown in table 2.

Table 2: Experimental design (Media-2 Paper-pulp based media)

\begin{tabular}{|c|c|c|c|}
\hline Factor \& Symbol & Level 1 & Level 2 & Level 3 \\
\hline Abrasive mesh size (A) & 120 & 220 & 320 \\
\hline \% Abrasive con. (B) & 33 & 50 & 66 \\
\hline \% Liquid synthesizer (C) & 30 & 40 & 50 \\
\hline
\end{tabular}

The nine samples of rice husk ash-based AFM media is prepared for the rheology study of media are shown in figure 9.

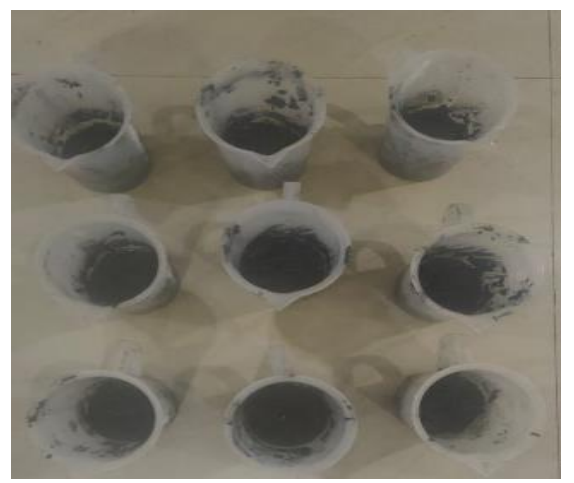

Figure 9. Samples of rice husk ash-based AFM media.

The viscosity responses of rice husk ash-based AFM media are shown in table 3.

Table 3. The Plan of experiment and experimental results for the viscosity of EFSAFM (Rice husk ash-based AFM media)

\begin{tabular}{|c|c|c|c|c|}
\hline $\mathbf{S}$ & Abrasive Mesh & $\begin{array}{c}\text { \% Abrasive } \\
\text { Concentration }\end{array}$ & $\begin{array}{c}\text { \% Liquid } \\
\text { Synthesizer }\end{array}$ & $\begin{array}{c}\text { Viscosity (Pa- } \\
\text { Sec) }\end{array}$ \\
\hline 1 & 120 & 33 & 30 & 2.50 \\
\hline 2 & 120 & 50 & 40 & 2.80 \\
\hline 3 & 120 & 67 & 50 & 3.10 \\
\hline 4 & 220 & 33 & 40 & 2.90 \\
\hline 5 & 220 & 50 & 50 & 2.20 \\
\hline
\end{tabular}




\begin{tabular}{|c|c|c|c|c|}
\hline 6 & 220 & 67 & 30 & 2.58 \\
\hline 7 & 320 & 33 & 50 & 2.40 \\
\hline 8 & 320 & 50 & 30 & 2.68 \\
\hline 9 & 320 & 67 & 40 & 2.80 \\
\hline
\end{tabular}

Upon experimental design analysis using minitab19 statistical software, it can be reported from the main effects plot for SN ratios for larger is better, as shown in figure 10, that the most influential AFM media parameter is liquid synthesizer followed by the percentage of abrasive concentration, and abrasive mesh size which affects the media viscosity. Here, the concentration of media was 120 mesh size, 67\% abrasive concentration, and $40 \%$ liquid synthesizer within the considered range.

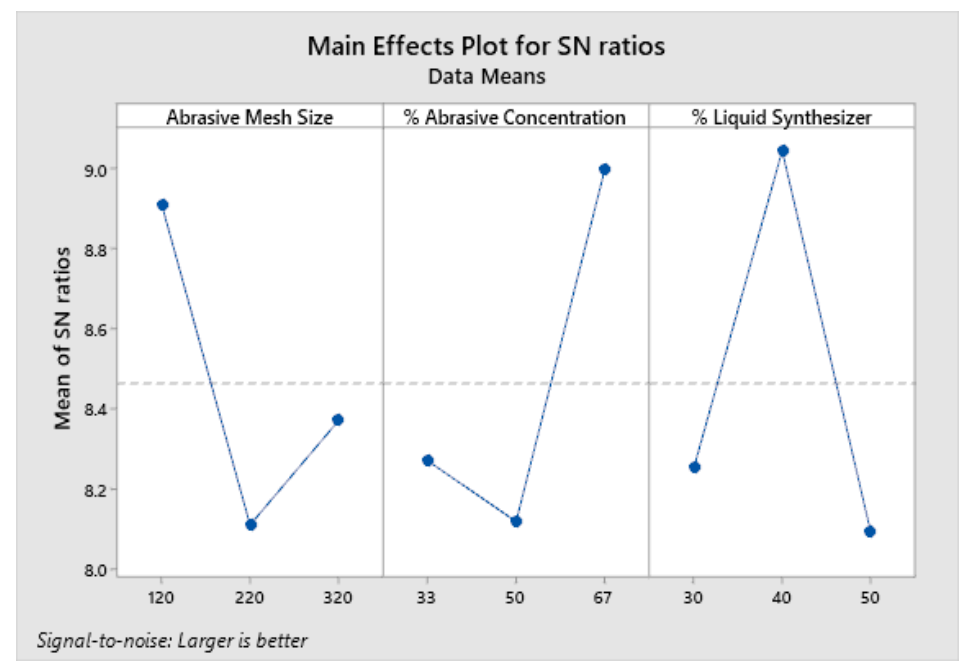

Figure 10. Main effects plots for SN ratios of AFM media viscosity.

\section{Results and Discussion}

The 3D printed samples of the hollow truncated cone are manufactured to be investigated the one-way AFM process parameters. The improvement of surface roughness was measured using Taylor Hobson's Talysurf® PRO surface roughness tester. In this study, the effects of AFM process parameters, i.e., media viscosity, layer thickness, and finishing time, are evaluated. An experimental design of Taguchi L9 orthogonal array was designed using 
Minitab 19 statistical software to analyze the AFM finishing process parameters. Table 3 shows that three process parameters like media viscosity, layer thickness, and finishing time are considered as control factors for the experimentation.

Table 3. Process Parameters and their levels for experimental design (finishing of FDM printed hollow truncated cone shape)

\begin{tabular}{|c|c|c|c|}
\hline Parameters & Level 1 & Level 2 & Level 3 \\
\hline Media viscosity (Pa-sec) & 2.50 & 2.80 & 3.10 \\
\hline \% Layer thickness & 0.1 & 0.2 & 0.3 \\
\hline Finishing Time (Min) & 30 & 45 & 60 \\
\hline
\end{tabular}

Each parameter has a 3-levels, namely, low, medium, and high. The \% improvement of surface quality, i.e., reduction in Ra of FDM printed hollow truncated cone shape, is shown in table 4.

Table 4. Design for experimentation of surface improvement on FDM printed hollow truncated cone shape

\begin{tabular}{|l|l|c|c|c|c|l|}
\hline S. & \multicolumn{1}{|c|}{ Media } & Layer & Finishing & Initial Surface & Post finishing & Improvement \\
N. & Viscosity & Thickness & Time (Min. $)$ & Roughness & Surface \\
$(\mathrm{mm})$ & & $\left(\mathrm{R}_{\mathrm{a}}\right.$ in $\left.\mu \mathrm{m}\right)$ & Roughness \\
$\left(\mathrm{R}_{\mathrm{a}}\right.$ in $\left.\mu \mathrm{m}\right)$ & \\
\hline 1 & 2.5 & 0.1 & 30 & 16.40 & 11.66 & 30.77 \\
\hline 2 & 2.8 & 0.2 & 45 & 23.20 & 14.70 & 38.28 \\
\hline 3 & 3.1 & 0.3 & 60 & 27.20 & 14.68 & 47.78 \\
\hline 4 & 2.5 & 0.2 & 60 & 22.50 & 11.80 & 49.76 \\
\hline
\end{tabular}




\begin{tabular}{|l|l|l|l|l|l|l|}
\hline 5 & 2.8 & 0.3 & 30 & 29.20 & 15.23 & 49.53 \\
\hline 6 & 3.1 & 0.1 & 45 & 15.50 & 4.20 & 77.93 \\
\hline 7 & 2.5 & 0.3 & 45 & 28.60 & 8.26 & 73.69 \\
\hline 8 & 2.8 & 0.1 & 60 & 14.60 & 6.26 & 61.32 \\
\hline 9 & 3.1 & 0.2 & 30 & 21.93 & 0.88 & 95.98 \\
\hline
\end{tabular}

The data analysis of the above experimental design of finishing process using minitab19 statistical software can be reported from the main effects plot for SN ratios for improvement of surface roughness keeping the ratio larger is better, as shown in figure 11, that the most influential finishing parameter is media viscosity followed by the finishing time and layer thickness. Here, the optimum parameters were observed at the 3.1 pa-sec media viscosity, 45 min. of finishing time and 0.2 layer thickness.

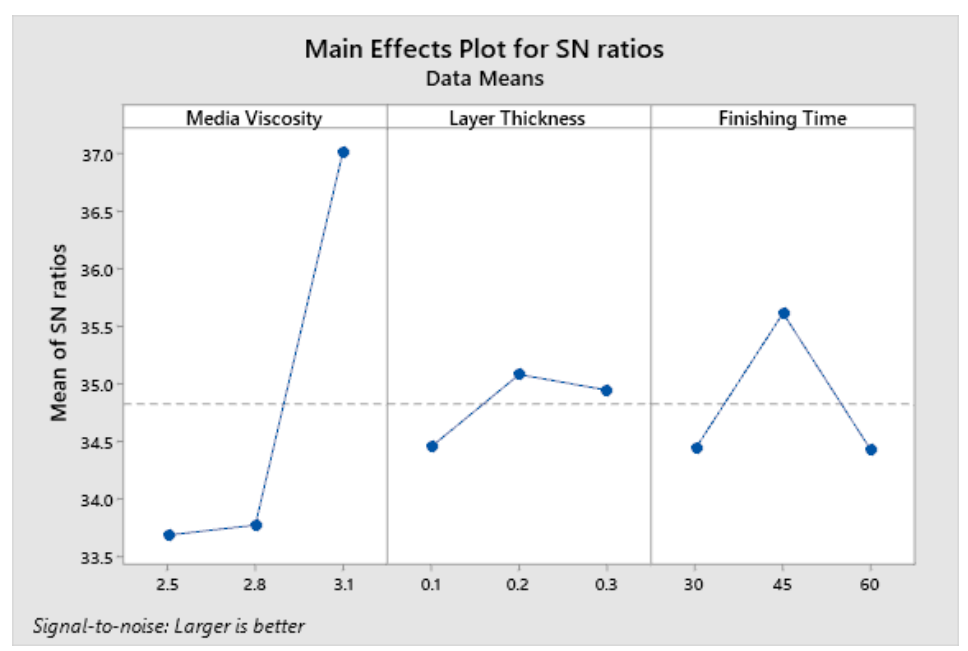

Figure 11. Main effects plots for SN ratios of finishing of FDM printed parts. 
Effect of media viscosity: AFM media viscosity is the main influential parameter during finishing FDM printed hollow truncated shape. As the improvement of surface roughness is increased by increasing the AFM media viscosity. It has happened because increased viscosity increases the shear force during the finishing operation.

Effect of finishing time: Finishing time is the second most influential parameter during finishing FDM printed hollow truncated shape. As the improvement of surface roughness is increased by increasing the finishing time up to the optimum value of $45 \mathrm{~min}$. It has happened because increased finishing time at optimum level reduces the surface roughness increases at maximum level. After the optimum level of finishing time, the reduction surface roughness was fixed at maximum percentage improvement.

Effect of layer thickness: Layer thickness of FDM printed parts is the third least influential parameter during finishing FDM printed hollow truncated shape. As the maximum improvement of surface roughness was found at 0.2 layer thickness of the workpiece. It is because of the influence of the initial value of surface roughness.

\section{Conclusions and Future Scopes}

The present experimental investigation study has opened the path for a better understanding of the AFM process's parametric dependence on improving surface roughness of FDM printed hollow truncated cone shape. The optimization of media viscosity is the essential parameter during the internal finishing of the FDM printed hollow truncated cone surface. The optimum viscosity was found at the following constituents of AFM finishing media: 120 abrasive mesh size, 67 percent of abrasive concentration, and 45 percent of liquid synthesizer. The experimental investigation of the one-way AFM process is showed that media viscosity and finishing time are the most influential process parameters. The maximum improvement was found at the following process parameter of one-way AFM: 3.10 Pa-sec media viscosity, 
$30 \mathrm{~min}$. of finishing time, and 0.2 layer thickness of the workpiece. The initial surface roughness was reduced from $21.93 \mu \mathrm{m}$ to $0.88 \mu \mathrm{m}$ by $95.98 \%$ improvement. To further understand, model, and simulate the AFM process to finish FDM printed parts, the flow of AFM media may be examined using the computational fluid dynamics simulation technique to investigate the flow characteristics of AFM media.

\section{Conflict of Interest}

The authors declare that they have no conflict of interest.

\section{Acknowledgment}

The authors would like to thank the Science and Engineering Research Board, Department of Science and Technology, Government of India for supporting this work through the grant DST-SERB EMR/2016/003372.

\section{References}

Ali, P. et al. (2020) 'Material removal analysis of hybrid EDM-assisted centrifugal abrasive flow machining process for performance enhancement', undefined [Preprint]. Available at: https://www.semanticscholar.org/paper/Material-removal-analysis-of-hybrid-EDM-assistedAli-Walia/fcf01761d2c2e7ac5db54cdfcc79025aa51855de (Accessed: 21 November 2021).

A. Wahab Hashmi, H. Singh Mali, and A. Meena (2021), "Improving the surface characteristics of additively manufactured parts: A review," Mater. Today Proc., p. S2214785321031436, May 2021, doi: 10.1016/j.matpr.2021.04.223.

A. W. Hashmi, H. S. Mali, and A. Meena (2021), "The Surface Quality Improvement Methods for FDM Printed Parts: A Review," Fused Depos. Model. Based 3D Print., pp. 167194, 2021, doi: 10.1007/978-3-030-68024-4_9. 
A. W. Hashmi, D. H. S. Mali, and A. Meena (2020), "SURFACE QUALITY IMPROVEMENT METHODS OF ADDITIVELY MANUFACTURED PARTS: A REVIEW," Solid State Technol., vol. 63, no. 6, Art. no. 6, Dec. 2020.

Anggraeni, S., Nandiyanto, A.B.D., Putri, S.R., Anshar, A.N., Maulana, A., Nurazizah, S., Nurjihan, Z., et al. (2021), "Mechanical Properties of briquette by mixing rice and micrometer-sized carbon particles from potato and yam skins", Journal of Engineering Research, pp. 1-15.

Arivazhagan Pugalendhi*, SenthilMurugan Arumugam, Rajesh Ranganathan, S.G. (2021), "3D printed patient-specific bone models for anatomy education from medical imaging", Journal of Engineering Research, No. 3, pp. 1-12.

Baraiya, R. et al. (2020) 'In-situ simultaneous surface finishing using abrasive flow machining via novel fixture', undefined [Preprint]. Available at: https://www.semanticscholar.org/paper/In-situ-simultaneous-surface-finishing-using-flowBaraiya-Babbar/521eeb85b6bae14d3245f6bc50548d0f3c10ddb4 (Accessed: 21 November 2021).

Dabrowski, L., Marciniak, M. and Szewczyk, T. (2006) 'Analysis of Abrasive Flow Machining with an Electrochemical Process Aid':, Proceedings of the Institution of Mechanical Engineers, Part B: Journal of Engineering Manufacture [Preprint]. doi:10.1243/095440506X77571.

Dixit, N., Sharma, V. and Kumar, P. (2021a) 'Development and characterization of xanthan gum-based abrasive media and performance analysis using abrasive flow machining', Journal of Manufacturing Processes, 67, pp. 101-115. doi:10.1016/j.jmapro.2021.04.053. 
Dixit, N., Sharma, V. and Kumar, P. (2021b) 'Experimental investigations into abrasive flow machining (AFM) of 3D printed ABS and PLA parts', Rapid Prototyping Journal [Preprint]. doi:10.1108/RPJ-01-2021-0013.

Jha, S. and Jain, V. (2004) 'Design and development of the magnetorheological abrasive flow finishing (MRAFF) process', undefined [Preprint]. Available at: https://www.semanticscholar.org/paper/Design-and-development-of-the-magnetorheologicalJha-Jain/793d41bf714a812a16896b42351d3e225ecbe693 (Accessed: 21 November 2021).

Kum, C. et al. (2020) 'Prediction and compensation of material removal for abrasive flow machining of additively manufactured metal components', undefined [Preprint]. Available at: https://www.semanticscholar.org/paper/Prediction-and-compensation-of-material-removalfor-Kum-Wu/784a3017a7860aa8b2289fcfa249bee7b3b6525f (Accessed: 11 November 2021).

Kumar, S.S. and Hiremath, S.S. (2016) 'A Review on Abrasive Flow Machining (AFM)', Procedia Technology, 25, pp. 1297-1304. doi:10.1016/j.protcy.2016.08.224.

Kumar, H., Sharma, A., Shrivastava, Y., Khan, S.A. and Arora, P.K. (2021), "Optimization of process parameters of pin on disc wear set up for 3D printed specimens", Journal of Engineering Research (Kuwait), Vol. 9, pp. 133-145.

Lin, B. et al. (2020) 'Theoretical and experimental analysis of material removal and surface generation in novel fixed abrasive lapping of optical surface', Journal of Materials Processing Technology, 279, p. 116570. doi:10.1016/j.jmatprotec.2019.116570.

Mohamed, O.A., Masood, S.H. and Bhowmik, J.L. (2015), “Optimization of fused deposition modeling process parameters", Advances in Manufacturing, Vol. 3 No. 1, pp. 4253.

N, J., G, S. and A D, P. (2021), "Effect of Printing Parameters of 3D Printed PLA Parts on 
Mechanical Properties”, Journal of Engineering Research, pp. 1-16.

Perry, W. and Stackhouse, J. (1989) 'Gas Turbine Applications of Abrasive Flow Machining', undefined [Preprint]. Available at: https://www.semanticscholar.org/paper/GasTurbine-Applications-of-Abrasive-Flow-Machining-Perry-

Stackhouse/38847c3555ad6fe7d7e56b9a0c72e1932dba6022 (Accessed: 21 November 2021). Rajesha, S. et al. (2010) 'Performance study of a natural polymer based media for abrasive flow machining', undefined [Preprint]. Available at: https://www.semanticscholar.org/paper/Performance-study-of-a-natural-polymer-basedmedia-Rajesha-Venkatesh/61d00a3bb8207751d7e04f919ebea59832317cdf (Accessed: 21 November 2021).

Rhoades, L. (1991) 'Abrasive flow machining: a case study', undefined [Preprint]. Available at: https://www.semanticscholar.org/paper/Abrasive-flow-machining\%3A-a-case-studyRhoades/4ac332a08e33b109505fe99d0e69bcc31609ffd7 (Accessed: 21 November 2021).

Sankar, M.R. et al. (2019) 'Medium rheological characterization and performance study during rotational abrasive flow finishing (R-AFF) of $\mathrm{Al}$ alloy and $\mathrm{Al}$ alloy/SiC MMCs', The International Journal of Advanced Manufacturing Technology, 100(5), pp. 1149-1163. doi:10.1007/s00170-018-2244-y.

Shabgard/861c053f712f575fa60730a5c790c19bf8b750f5 (Accessed: 21 November 2021).

Singh, S. and Sankar, M.R. (2019) 'Development of polymer abrasive medium for nanofinishing of microholes on surgical stainless steel using abrasive flow finishing process':, Proceedings of the Institution of Mechanical Engineers, Part B: Journal of Engineering Manufacture [Preprint]. doi:10.1177/0954405419883768.

Sudhakara, D., Suresh, S. and Vinod, B. (2020) 'Experimental Study on Abrasive Flow Machining (AFM): New Approach for Investigation on Nano-SiC in the Improvement of 
Material Removal and Surface Finishing', Journal of Bio- and Tribo-Corrosion, 6(1), pp. 112. doi:10.1007/s40735-019-0321-X.

Walia, R.S., Shan, H. and Kumar, P. (2009) 'Modelling of centrifugal-force-assisted abrasive flow machining', undefined [Preprint]. Available at: https://www.semanticscholar.org/paper/Modelling-of-centrifugal-force-assisted-abrasiveWalia-Shan/d191af51ec105ef9d349682af75a7cbaf0d7c109 (Accessed: 21 November 2021). 